\title{
Multi-Slice to Volume Registration of Ultrasound Data to a Statistical Atlas of Human Pelvis
}

\author{
Sahar Ghanavati ${ }^{\mathrm{a}_{*}}$, Parvin Mousavi ${ }^{\mathrm{a}, \mathrm{b}}$, Gabor Fichtinger ${ }^{\mathrm{a}, \mathrm{b}, \mathrm{c}, \mathrm{d}}$, Pezhman Foroughi $^{\mathrm{c}}$ and Purang \\ Abolmaesumi ${ }^{\mathrm{a}, \mathrm{b}, \mathrm{d}, \mathrm{e}}$ \\ ${ }^{a}$ Dept. of Electrical and Computer Engineering, Queen's University, Kingston, ON, Canada \\ ${ }^{\mathrm{b}}$ School of Computing, Queen's University, Kingston, ON, Canada \\ ${ }^{\mathrm{c}}$ Dept. of Computer Science, Johns Hopkins University, Baltimore, MD, USA \\ ${ }^{\mathrm{d}}$ Dept. of Surgery, Queen's University, Kingston, ON, Canada \\ ${ }^{\mathrm{e}}$ Dept. of Electrical and Computer Engineering, University of British Columbia, Vancouver, BC, \\ Canada
}

\begin{abstract}
Identifying the proper orientation of the pelvis is a critical step in accurate placement of the femur prosthesis in the acetabulum in Total Hip Replacement (THR) surgeries. The general approach to localize the orientation of the pelvis coordinate system is to use X-ray fluoroscopy to guide the procedure. An alternative can be employing intra-operative ultrasound (US) imaging with pre-operative CT scan or fluoroscopy imaging. In this paper, we propose to replace the need of pre-operative imaging by using a statistical shape model of the pelvis, constructed from several CT images. We then propose an automatic deformable intensity-based registration of the anatomical atlas to a sparse set of 2D ultrasound images of the pelvis in order to localize its anatomical coordinate system. In this registration technique, we first extract a set of 2D slices from a single instance of the pelvic atlas. Each individual 2D slice is generated based on the location of a corresponding 2D ultrasound image. Next, we create simulated ultrasound images out of the 2D atlas slices and calculate a similarity metric between the simulated images and the actual ultrasound images. The similarity metric guides an optimizer to generate an instance of the atlas that best matches the ultrasound data. We demonstrated the feasibility of our proposed approach on two male human cadaver data. The registration was able to localize a patient-specific pelvic coordinate system with origin translation error of $2 \mathrm{~mm}$ and $3.45 \mathrm{~mm}$, and average axes rotation error of 3.5 degrees and 3.9 degrees for the two cadavers, respectively.
\end{abstract}

Keywords: Freehand ultrasound, Statistical anatomical atlas, Intensity-based registration, Deformable registration, Total Hip Replacement, Pelvis.

\section{INTRODUCTION}

The increase in the aging population, hence more prevalent osteoarthritis and degenerated hip joint cases, has made Total Hip Replacement (THR) a common surgical procedure. THR involves replacing the hip joint with a prosthetic implant, consisting of femoral and acetabular components. Misalignment of the acetabular component in this procedure can cause dislocation of the hip joint, impingement of the acetabulum and the need of a revision operation. Computer navigation systems can decrease the probability of a revision operation by precisely localizing the pelvic coordinate system and acetabular component orientation ${ }^{1}$. A navigation system which is commonly used in computer assisted orthopedic surgeries is the integration of pre-operative Computed Tomography (CT) and intra-operative X-ray imaging $^{2,3}$. A 2D/3D registration method using a pre-operative CT and a single standard intra-operative anteriorposterior radiograph has been proposed for evaluation of acetabular cup-orientation in THR by Zheng et al ${ }^{4}$. In addition to extra cost, the pre-operative imaging exposes the patient to significant amount of ionizing radiation. To eliminate the need for pre-operative data, a statistical deformable 2D/3D registration method is introduced to register a statistical

*sahar@cs.queensu.ca; Phone 16135336000 Ext. 74201

Medical Imaging 2010: Visualization, Image-Guided Procedures, and Modeling, edited by Kenneth H. Wong, Michael I. Miga, Proc. of SPIE Vol. 7625, 762500 - (c) 2010 SPIE · CCC code: 1605-7422/10/\$18 - doi: 10.1117/12.844080 
shape model of the pelvis constructed of several pelvic CT scans and the single intra-operative X-ray image ${ }^{5}$. However, the result is prone to error in a real-time procedure due to the static nature of X-ray imaging.

Ultrasound (US) imaging and ultrasound-based registration has been proposed as an alternative intra-operative imaging technique for computer-assisted orthopedic surgery, which benefits from the real-time and non-ionizing nature of ultrasound $^{6,7}$. Several approaches have been proposed to register freehand 2D ultrasound to CT images. A frame-byframe rigid registration method has been proposed by Peterhans et al. to register CT images to the ultrasound images during image acquisition ${ }^{8}$. This method estimates the rigid transform parameters by using an Unscented Kalman Filter (UKF). Barrat et al. have proposed a feature-based registration approach where both ultrasound and CT images are first automatically converted to probability maps that present the bone edge ${ }^{9}$. This approach is then used to register the point set extracted from the ultrasound to the bone surface in CT by optimizing the calibration parameters resulted from the tracked ultrasound acquisition.

Despite promising results above, it is still desirable to eliminate $\mathrm{CT}$ imaging and ionizing radiation exposure to the patient altogether. A solution is to replace CT with a statistical atlas model of the pelvis generated from a large population of patients. In a technique proposed by Foroughi et al. the pelvic bone surface is first segmented automatically from the ultrasound data using a dynamic programming approach ${ }^{10}$. The segmentation is then followed by the registration of the segmented points to a statistical atlas model ${ }^{11}$. The primary drawback of this technique is the need for the segmentation of the bone surface from ultrasound data that is generally a very challenging task. This is due to the existence of speckle and shadow in ultrasound images. This method also needs a good approximate initial alignment of the extracted points from the ultrasound with the mean shape of the atlas due to the high sensitivity of the point-based registration methods that use Iterative Closest Point (ICP) optimization, to the initialization. ${ }^{12}$

In this work, we propose a fully automated method for the registration of a statistical anatomical atlas to the ultrasound using an intensity-based registration. The proposed approach eliminates the need for the segmentation of the pelvis surface in the ultrasound and as a result, improves the accuracy of the registration. Our atlas generation method is based on the iterative method presented by Chintalapani et al. ${ }^{13}$. We introduce a two-step multi-slice to volume registration method in which, the mean shape of the atlas is first registered rigidly to the freehand 2D ultrasound images, based on an ultrasound simulation method proposed by Wein et al. ${ }^{14}$. Next, an instance of the atlas is created using the atlas dominant mode weights and the similarity metric is calculated between the actual ultrasound and the ultrasound images simulated from the instance of the atlas. The similarity measure is used to optimize the atlas variation mode weights, so that the optimized weights generate an instance of the atlas that best matches the ultrasound images and the patient specific anatomy.

\section{METHODS}

\subsection{Statistical Atlas of the Pelvis}

The statistical anatomical atlas that we used is a shape model generated from 110 pelvises from CT scans of healthy male patients. Our atlas consists of a volumetric tetrahedral mesh representing the mean shape (M) and shape variation modes $\left(\lambda_{i}\right)$. To construct the shape model, a CT image was chosen as the template. The bone in the template CT was segmented and a tetrahedral mesh was introduced on the bone. The other CTs were aligned with the template CT with a deformable registration method, resulting in a deformation field. A mesh instance was created for each of the CT images by interpolating the deformation field on vertices of the template mesh and a rigid registration was performed on all of the meshes. The mean shape and the variation modes were generated from Principal Component Analysis (PCA) on the registered mesh instances ${ }^{13}$. Once the atlas is created, new instances of the atlas can be generated as follows:

$$
\mathrm{M}^{*}=\mathrm{M}+\sum_{i=1}^{n} \alpha_{i} \lambda_{i}
$$

where $\mathrm{M}^{*}$ is the instance of the atlas, $\mathrm{M}$ is the mean shape, $n$ is the number of variation modes and $\lambda_{\mathrm{i}}$ and $\alpha_{\mathrm{i}}$ are the $i$ th mode of variation and its corresponding weight, respectively. The minimum number of variation modes required to 
reconstruct any given pelvis shape is 15 dominant modes, according to Chintalapani et al. ${ }^{13}$. For our atlas generation, we also chose 15 dominant principal modes which represent a shape variation range of $75.9 \%$.

In this atlas generation method, the mean shape and all the variations of the atlas are represented by a volumetric tetrahedral mesh. In order to perform the registration between the atlas and the US images, we performed an interpolation on the tetrahedral mesh for each atlas instance to generate a volumetric data. This interpolation was done after atlas instantiation during the registration process. For our US simulation-based registration it is enough to have a

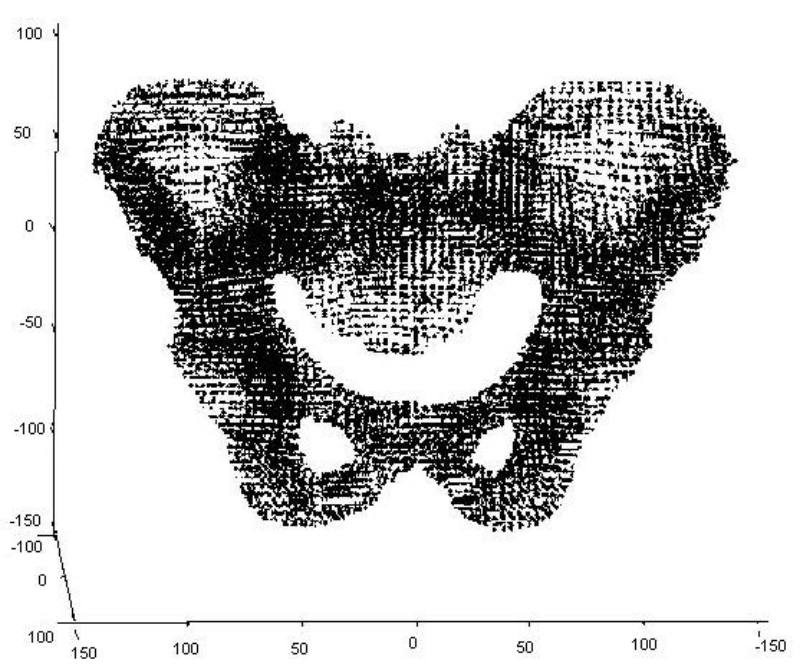

(a)

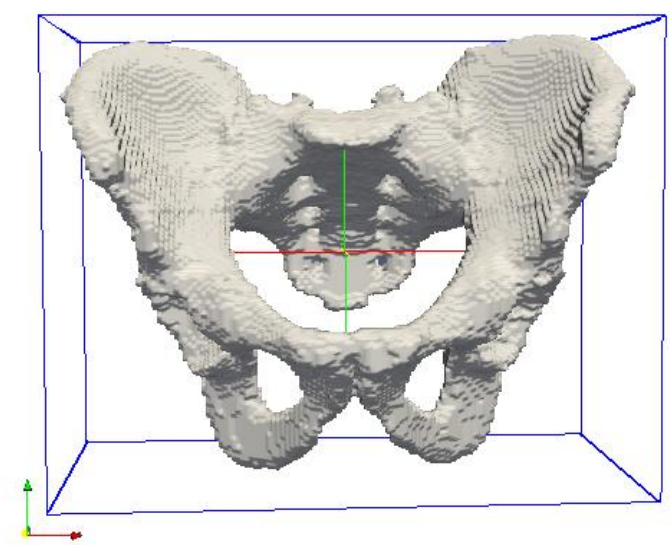

(b)

Fig. 1. a) The point representation of the vertices in mean shape and b) the interpolated volume.

binary atlas instance. We assumed that the intensity of the bone for the interpolated volumetric atlas instance is 1024 which is the average bone intensity in CT images. The intensity of the voxels surrounding the bone were set to zero which is assumed to be the intensity of soft tissue in CT images. Assigning different intensities to different regions in the atlas instance image results in the US simulation slices having a similar intensity distribution as the actual US images, since the intensity of soft tissue surrounding the bone in the US image is significantly different from the pixels of the shadow region beneath the bone surface. The tetrahedral mesh and the volumetric representation of the mean shape are shown in Figure 1.

\subsection{US Image Acquisition}

A stack of ultrasound slices were captured from the right and left iliac crest of the pelvis including the anterior superior iliac spines and also from the body of the pubis containing pubic tubercles (Figure 2), where landmarks can be located to define the coordinate system of the pelvis ${ }^{15}$. The ultrasound images were rendered by a tracked freehand 2D transducer. We chose a set of slices that had an acceptable image quality and bone visibility and their corresponding tracking transformations from all the acquired US images and used them in the registration process.

\subsection{Multi-Slice to Volume Registration}

We proposed a multi-slice to volume registration method to register the atlas to the coordinate system of the ultrasound. The registration process consists of a rigid registration of the atlas mean shape and as a result all the variations of the atlas, to the US images. This is followed by a deformable registration to calculate the variation mode weights that instantiate an atlas which best matches the pelvis anatomy represented by the ultrasound images (Figure 3). Instead of reconstructing an ultrasound volume from the 2D ultrasound images of the pelvis, we used the US tracking transformations to extract 2D slices from the atlas instance. The translation parameters in the transformation identify 
the position of the slice to be extracted from the volume and the rotation parameters indicate the orientation of the 2D slices. Then the corresponding atlas instance and ultrasound slices with the same tracking transformations were compared in a fashion that will be explained, to find the similarity measure in the registration process.

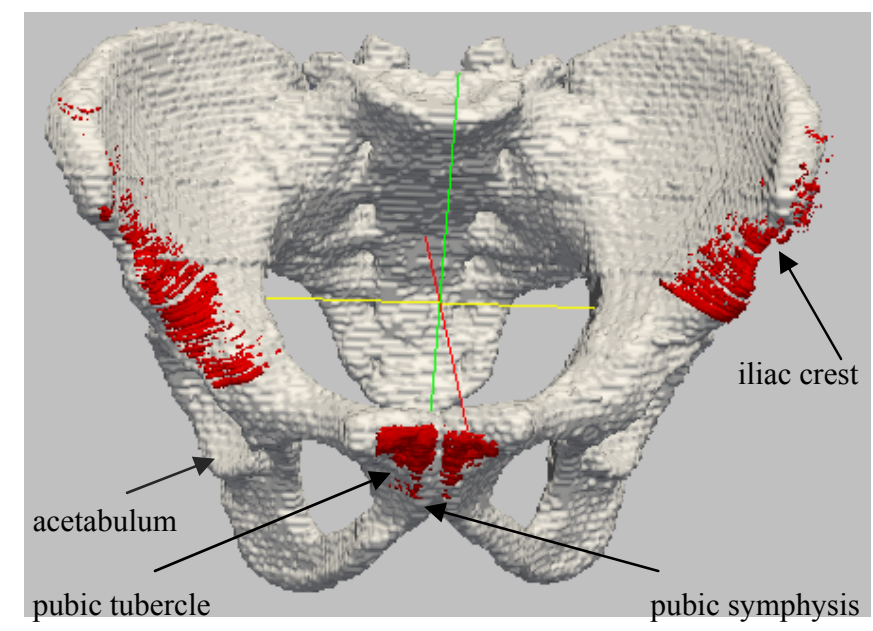

Fig. 2. The ultrasound acquisition regions (right and left iliac crest and the pubis) are marked red on the pelvis.

\subsubsection{Ultrasound Simulation}

As we explained in Section 2.1, we assigned intensities close to that of the CT images to the atlas instance volumes. Because of the tissue occlusion in the US images beneath the bone edge, the CT-like images extracted from atlas instance volumes should have been modified so that a rational comparison between each pair of atlas slices and US images would be possible. We used the ultrasound simulation approach proposed by Wein et al. ${ }^{14}$ to measure the similarity between the extracted atlas slices and the ultrasound slices. Since our atlas intensities did not contain the intensity information of an actual CT orthopaedic image (CT Hounsfield unit and tissue echogeneity), the ultrasound simulation procedure we employed was simpler and thus faster than Wein's approach.

To simulate an ultrasound image from each slice extracted from an atlas instance, we first located three regions in the slice: pixels above the bone associated to the soft tissue, the bone edge and the shadow region associated with the bone itself. For the first region, intensity values similar to the US were computed by averaging the intensity of pixels in the pair US image that correspond to the region above the bone in the extracted slice. The maximum intensity in the US image was used as the intensity of simulated-US pixels that correspond to the bone edge in the atlas instance slice. Finally, the shadow intensity for the simulated-US was calculated by averaging the intensity of US pixels corresponding to the region beneath the bone in the atlas instance slice. A slice extracted from an atlas instance, its simulated ultrasound image and the corresponding US image are shown in Figures 3 and 4.

\subsubsection{Rigid Registration}

In order to initialize the rigid registration, we aligned the mean shape close to the US coordinate system manually resulting in all instances of the atlas being transferred to this initial position. The registration is then started by extracting 2D slices from the mean shape volume using the ultrasound-tracked transformations. A simulated US was created from each of the slices extracted from the atlas instance as described in Section 2.3.1. The similarity metric was then calculated between the actual ultrasound images and the simulated ultrasound slices. The Linear Correlation of Linear Combination $\left(\mathrm{LC}^{2}\right)$ similarity metric is used, 


$$
\mathrm{LC}^{2}=1-\frac{\sum(U S(x, y)-\operatorname{Sim} U S(x, y))^{2}}{N * \operatorname{Var}(U S)}
$$

where $U S(x, y)$ and $\operatorname{Sim} U S(x, y)$ are the actual and simulated US image pixels' intensities, respectively. $N$ is the number of overlapping pixels in ultrasound and simulated ultrasound images and $\operatorname{Var}(U S)$ is the variance of US image pixels intensities. The similarity metric led the optimizer to update the registration rigid parameters and the mean shape was transformed using the rigid transformation. The slice extraction and US simulation was repeated until the rigid parameters were optimized to the best value that gives the best position for mean shape with respect to US images. To guarantee the robustness of the registration we used Covariance Matrix Adaptation - Evolution Strategy (CMA-ES) as the optimizer ${ }^{16,17}$. The result of this registration was an Euler 3D transformation with which the mean shape and all the instances of the atlas were transformed to be in correct rigid alignment with the US images for the deformable registration.

\subsubsection{Deformable Registration}

Deformable registration of the atlas to ultrasound images was an iterative optimization approach (Figure 3), similar to the rigid registration process except for the registration parameters. The registration parameters are the weights for the most dominant atlas shape variation modes. The registration is comprised of the following steps:

1) A new instance of the atlas was generated by adjusting the weights associated with its principal variation modes. We used the 15 most dominant modes of variation of the atlas for a precise shape recovery. To create an instance of the atlas that best fits a patient, the weights associated with the variation modes were optimized during the registration process.

2) $2 \mathrm{D}$ slices were extracted from the current atlas instance based on the US tracked transformations and a simulated ultrasound was created from each of the slices.

3) The $\mathrm{LC}^{2}$ similarity measure was calculated between the simulated and the actual ultrasound slices.

4) The CMA-ES optimizer used the similarity metric criteria to update the atlas shape weighting parameters and the previous steps were repeated.

These steps were repeated until the similarity measure converged to its best value and the weights associated with the anatomical shape of the US data were retrieved.

\section{RESULTS AND DISCUSSION}

We examined our proposed registration method on two male human cadavers, neither of which were involved in the atlas generation. The test subjects were previously used to test the registration method presented in [11]. Ultrasound images were rendered from the test subjects using SonoSite portable ultrasound system (SonoSite Inc., Bothell,WA) with a high frequency transducer and a data collection rate of 10 frames per second. The ultrasound pixel size was 0.1 $\mathrm{mm}$. The ultrasound probe was tracked by a Polaris optical tracking system (Northern Digital Inc., Waterloo, Canada) and was calibrated by the standard crosswire technique ${ }^{18}$. All the registrations were programmed in $\mathrm{C}++$ language using Insight Segmentation and Registration Toolkit (ITK) and were performed on an Intel Q6600,with $2 \times 2.40 \mathrm{GHz}$ Quad-core CPU and 3.24 GB of RAM.

To evaluate the accuracy of our registration process a co-registered CT image to the US slices was used as the ground truth. The CT volume consisted of 260 slices, each containing 256 by 256 pixels with a slice thickness of $1 \mathrm{~mm}$ and pixel spacing of $0.7 \mathrm{~mm}$. To register the CT volume to the ultrasound coordinate system without having fiducial markers, the bone surface was segmented in both US and CT. Sample points were extracted from the segmented bone surface in both images and the Iterative Closest Point (ICP) method $^{19}$ was performed on the sample points to compute 


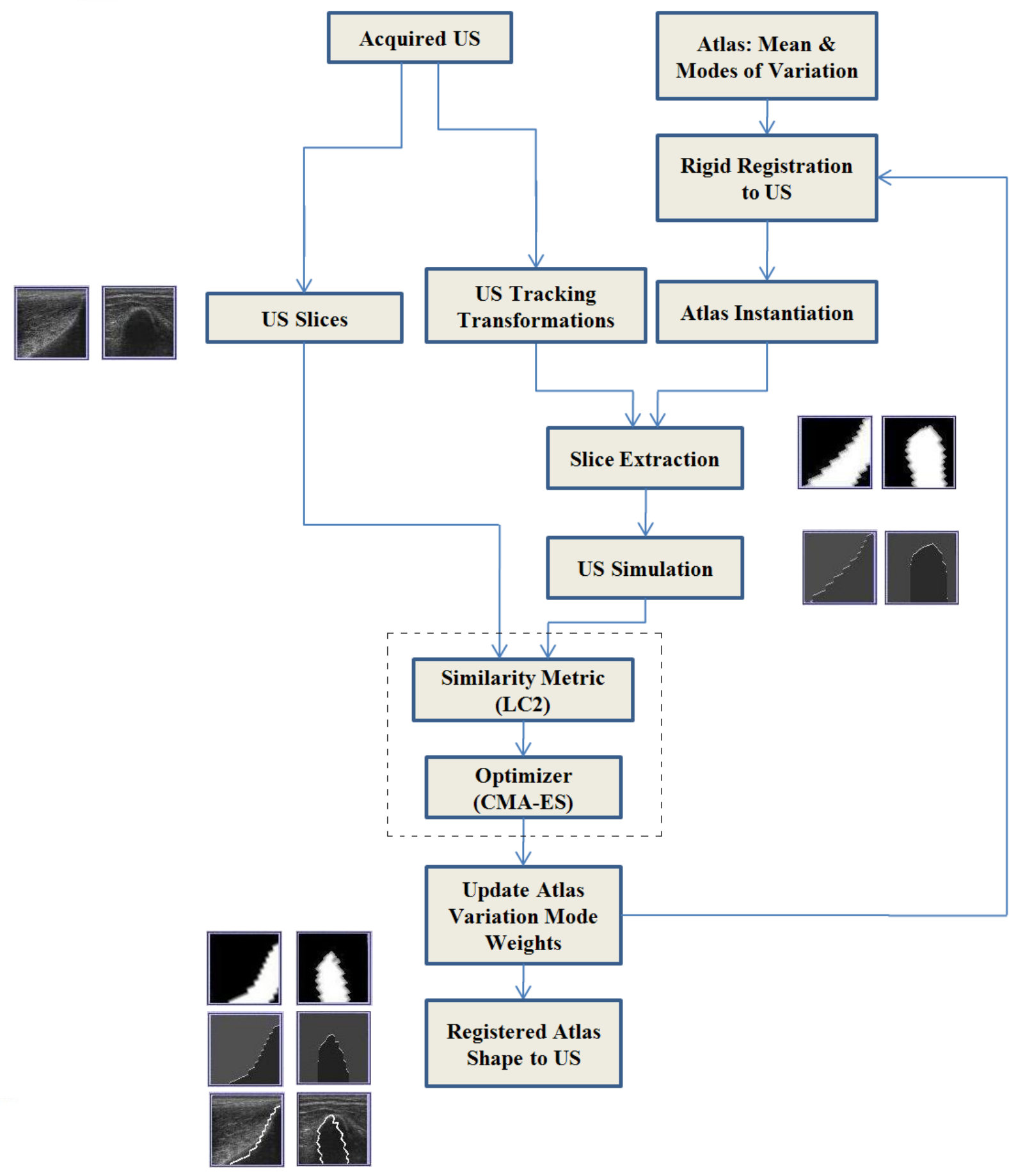

Fig. 3. Workflow for atlas to ultrasound registration. 
the rigid transformation parameters. The CT volume was then aligned with the US coordinate system using this transformation.

We evaluated the accuracy of our proposed multi-slice to volume registration method by calculating the Target Registration Error (TRE), which is the average distance error of four pelvic anatomical landmarks ${ }^{15}$ on the atlas instance registered to the ultrasound and the corresponding landmarks on the co-registered CT volume. In order to find the corresponding landmark coordinates, we first selected four anatomical landmarks on the mean shape manually. Then, the landmarks were transformed to CT coordinate system by registering the atlas to the CT. The coordinates of mean shape landmarks were also localized on the atlas instance which was registered to the ultrasound. The calculated TRE of the landmarks for the two test subjects are $9.15 \mathrm{~mm}$ and $7.21 \mathrm{~mm}$, respectively. The anatomical coordinate system of the pelvis was defined for the registered atlas using these landmarks and was compared to the CT coordinate system. The translation error of the origin and the rotation error of pelvis coordinate system axes for the cadavers are reported in table 1 . The corresponding registered atlas instance to ultrasound, the simulated ultrasound and the actual ultrasound slices and their overlays before and after registration are shown in Figure 4.

Table 1. Estimated error of the pelvis anatomical coordinate system of the registered atlas to ultrasound compared to the ground truth coordinate system of pelvis in the co-registered CT.

\begin{tabular}{|c|c|c|c|c|c|c|}
\hline Experiments & $\begin{array}{c}\text { translation } \\
\text { error } \\
\mathrm{x}(\mathrm{mm})\end{array}$ & $\begin{array}{c}\text { translation error } \\
\mathrm{y}(\mathrm{mm})\end{array}$ & $\begin{array}{c}\text { translation error } \\
\mathrm{z}(\mathrm{mm})\end{array}$ & $\begin{array}{c}\text { rotation error } \\
\alpha(\text { degree })\end{array}$ & $\begin{array}{c}\text { rotation error } \\
\beta(\text { degree })\end{array}$ & $\begin{array}{c}\text { rotation error } \\
\lambda(\mathrm{degree})\end{array}$ \\
\hline Cadaver 1 & 0.21 & 1.53 & 1.27 & 2.75 & 2.76 & 5.04 \\
\hline Cadaver 2 & 2.12 & 1.35 & 2.37 & 5.11 & 2.71 & 4.05 \\
\hline
\end{tabular}

It can be seen from Table 1 that despite the relatively high registration error of the anatomical landmarks (TRE), a precise localization of pelvis coordinate system was possible. Comparing the coordinate system estimation error with previous work presented in [11] shows that the origin translation error is comparable. But the rotation error of the coordinate system axes is higher specifically along $\mathrm{z}$ direction. The higher rotation error is a result of poor quality of ultrasound images acquired from the cadavers especially in the pubic tubercles region (Figure 5). Our registration method depends on an acceptable high intensity contrast of soft tissue and shadow region. As a result, the poor quality of acquire ultrasound images resulted in higher registration error and thus, higher error in coordinate system estimation compared to previous work presented in [11] which is based on bone surface segmentation. The ultrasound images are good enough to segment the bone surface precisely but the shadow region is poorly represented in ultrasound images of the cadavers which result in higher registration error in our method. The coordinate system origin was estimated based on the landmarks on the right and left iliac crest and the acceptable quality of the ultrasound images acquired from these regions resulted in an accurate estimation of the pelvis coordinate system origin. However, the poor quality of the ultrasound images acquired from the pubic tubercles caused a higher TRE for the pubic symphysis landmarks and as a result, higher rotation error along $\mathrm{z}$ axis of the estimated coordinate system for the registered atlas to the ultrasound.

The registration error is also partly caused by the ICP gold standard registration of the ultrasound and the CT, as well as the error present in atlas reconstruction of the anatomical shape of the test subjects that were not involved in the atlas generation (the error of atlas to CT registration). The atlas to CT registration error can be decreased by using more atlas variation modes in the registration.

\section{CONCLUSION AND FUTURE WORK}

In this paper, we introduced a fully automated registration method of a statistical anatomical atlas to tracked ultrasound images of the pelvis. By using this method, a framework for computer-guided surgery is possible which is only based on ultrasound imaging. Using an atlas in the framework will compensate for the missing anatomical information of the bone in US images. This method can eliminate the need of pre-operative imaging for total hip replacement. The registration is fully automated and no segmentation is needed in the process. This will increase the accuracy and 
reliability of this registration method in comparison with the previous methods introduced for registering the ultrasound to a shape model that use segmentation of the bone surface in the ultrasound. The registration is only using a small set of ultrasound slices rendered from left and right iliac crest of the pelvis and the pubic tubercles, which speeds up the data acquisition and registration significantly. This can eliminate the need for the reconstruction of a 3D ultrasound volume from freehand 2D images, which is a time consuming procedure. We have tested our proposed method on two male cadavers and have shown that the anatomical coordinate system of pelvis can be localized using the ultrasound images and the atlas anatomical coordinate system. Although our preliminary results show higher error than previous methods involving segmentation of the bone surface, the error is still in an acceptable range.
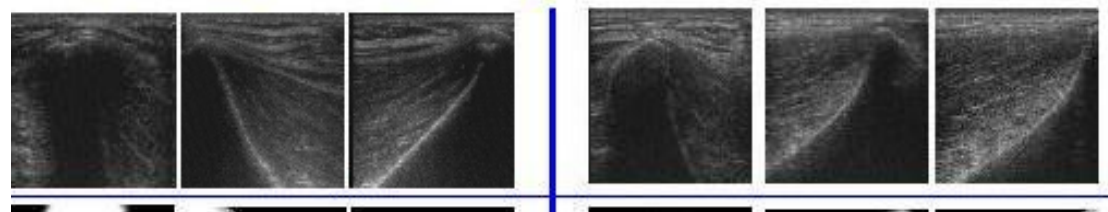

Ultrasound
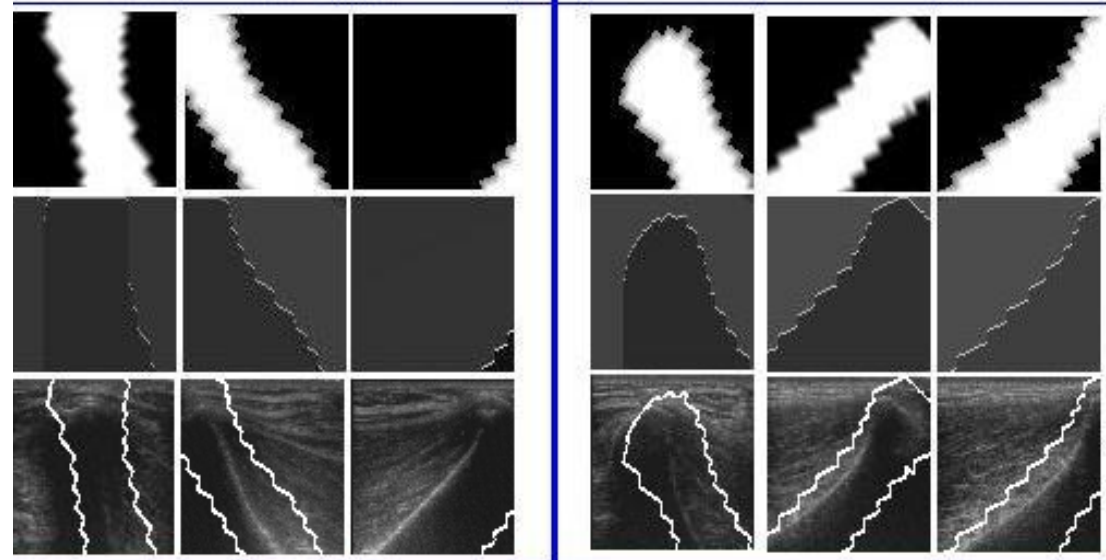

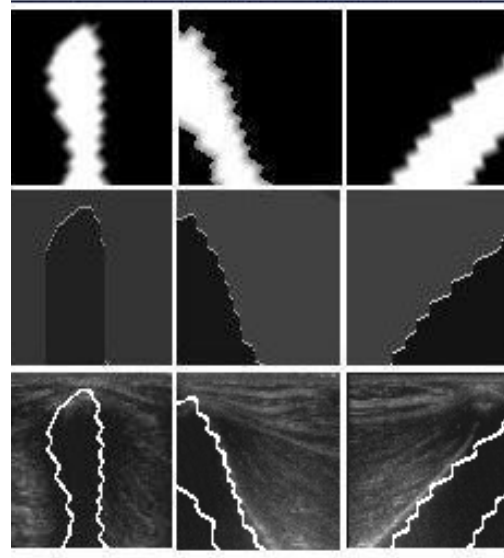

Cadaver 1
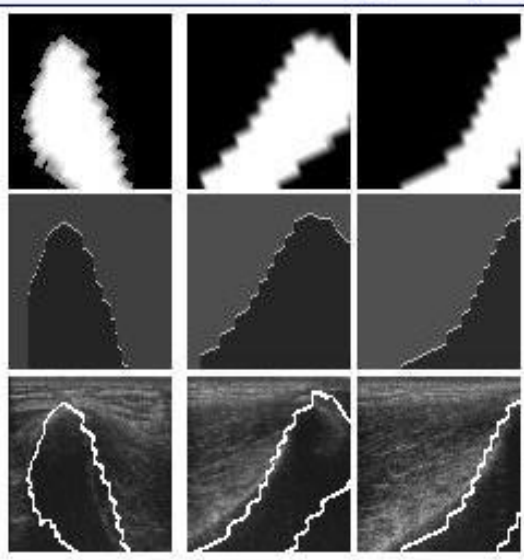

Cadaver 2
Simulated US

Before

Atlas Mean Shape

Registration

Mean Shape and US

Overlay

Simulated US

Registered Atlas and US

Overlay

Fig. 4. The US images, atlas extracted slices, the simulated US and the atlas and US slice overlays from different regions of the pelvis before and after registration.

In our future work, we will perform a patient study on candidates for hip arthroplasty to validate our method in a real scenario. We will investigate the practical usability of the atlas with the anatomical coordinate system stored in the mean shape, by examining the accuracy of the estimated anatomical coordinate system in the patient specific atlas shape compared to patient's pelvic coordinate system. We expect that the high quality ultrasound images from the patients 
would result in much lower errors than the cadaver study we presented in this paper. We will also test the capability of the atlas generated from healthy pelvises to localize the pelvic anatomical coordinate system of patients with hip disease and look into the effects of the local joint disease on the accuracy of anatomical coordinate system estimation. In addition, the atlas will be examined on female pelvises to evaluate the capability of our male atlas in eliminating the need for generating a unisex atlas composed of male and female pelvises. Although the registration of the male atlas to the female pelvis might not be precise, the coordinate system localization can still be accurate enough. We will also implement our method on GPU to decrease the computation time by adopting multithreading. ${ }^{20}$ This will help make the registration suitable for real-time performance.
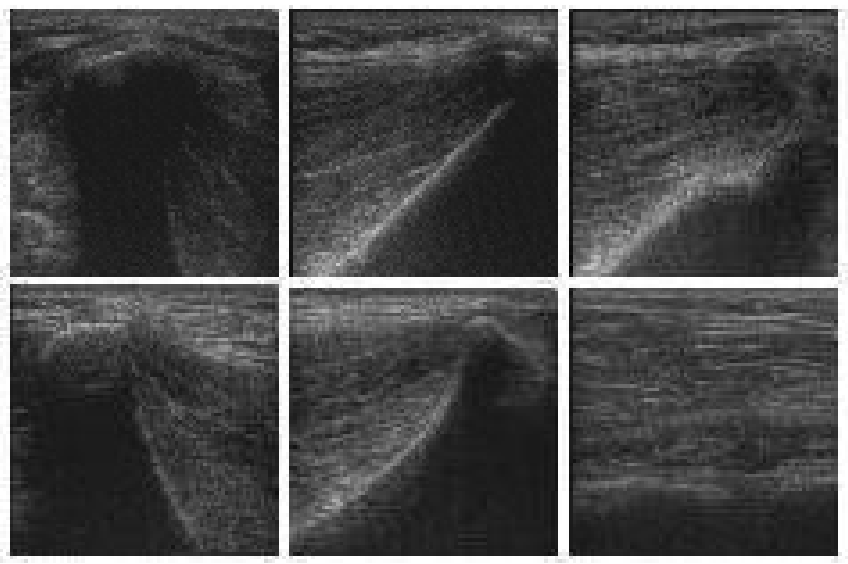

Fig. 5. Comparison of soft tissue and shadow region intensities in the ultrasound images of cadaver 1 (above) and cadaver 2 (below). The right column shows the poor quality of ultrasound images acquired from the pubic tubercles compared to ultrasound images from the right and left iliac crests (middle and left column.)

\section{ACKNOWLEDGMENTS}

This research is supported partly by the Natural Sciences and Engineering Research Council (NSERC) and the Canadian Institutes of Health Research (CIHR). The authors would like to also thank Dr. Ofri Sadowsky and Professor Russell Taylor of Johns Hopkins University for providing us with the pelvis atlas data needed for this research.

\section{REFERENCES}

[1] Honl, M., Schwieger, K., Salineros, M., Jacobs, J., Morlock, M., and Wimmer, M., "Orientation of the Acetabular Component: A Comparison of Five Navigation Systems with Conventional Surgical Technique," Journal of Bone and Joint Surgery 88 B (10), 1401-1405 (2006).

[2] Hamadeh, A., Lavallee, S. and Cinquin, P., "Automated 3-Dimentional Computed Tomographic and Fluoroscopic Image Registration," Computer Aided Surgery, 3, 11-19 (1998).

[3] Taylor, R. H., Joskowicz, L., Williamson, B., Gueziec, A., Kalvin, A., Kazanzides, P., Van Vorhis, R., Yao, J., Kumar, R., Bzostek, A., Sahay, A., Borner, M. and Lahmer, A., "Computer-integrated Revision Total Hip Replacement Surgery: Concept and Preliminary Results," Medical Image Analysis 3, 3, 301-319 (1999).

[4] Zheng, G., Steppacher, S., Zhang, X. and Tannast, M., "Precise Estimation of Post-operative Cup Alignment from Single Standard X-ray Radiograph," Medical Image Computing and Computer Assisted Intervention (MICCAI), Part II. LNCS 7492, 951-959 (2007).

[5] Zheng, G., "Statistically Deformable 2D/3D Registration for Accurate Determination of Post-operative Cup Orientation from Single Standard X-ray Radiograph," Medical Image Computing and Computer Assisted Intervention (MICCAI), Part I, LNCS 5761, 820-827 (2009). 
[6] Tonetti, J., Carrat, L., Blendea, S., Merloz, P., Troccaz, J., Lavallee, S. and Chirossel, J. P., "Clinical Results of Percutaneous Pelvic Surgery. Computer Assisted Surgery Using Ultrasound Compared to Standard Fluoroscopy," Computer Aided Surgery, 6, 204-211 (2001).

[7] Tonetti, J., Carrat, L., Lavallee, S., Pittet, L., Cinquin, P., Merloz, P. and Chirossel, J. P., "Percutaneous Iliosacral Screw Placement Using Image Guided Techniques," Clinical Orthopaedics and Related Research, 354, 103-110 (1998).

[8] M. Peterhans, H. Talib, M. G. Linguraru, M. Styner, M. A. G. Ballester, "A Method for Frame-by-Frame US to CT Registration in a Joint Calibration and Registration Framework," IEEE Biomedical ImagingSymposium, 1131-1134 (2008).

[9] D. Barratt, G. Penney, C. Chan, M. Slomczykowski, T. Carter, P. Edwards, and D. Hawkes, "Self-calibrating 3Dultrasound-based Bone Registration for Minimally Invasive Orthopedic Surgery," IEEE Transactions on Medical Imaging, vol. 25, no. 3, 312-23 (2006).

[10] P. Foroughi, E. Boctor, M. J. Swartz, R. H. Taylor, G. Fichtinger, "Ultrasound Bone Segmentation Using Dynamics Programming," IEEE Ultrasonics Symposium, 2523-2526 (2007).

[11] P. Foroughi, D. Song, G. Chintalapani, R. H. Taylor, G. Fichtinger, "Localization of Pelvic Anatomical Coordinate System Using US/Atlas Registration for Total Hip Replacement," D. Metaxas et al. (eds.) Medical Image Computing and Computer Assisted Intervention (MICCAI), Part II, LNCS 5242, 871-879. Springer, Heidelberg (2008).

[12] P. Foroughi, R. H. Taylor, G. Fichtinger, "Automatic Initialization for 3D Bone Registration," Proceedings of SPIE Medical Imaging, Vol. 6918, 69182P, 2523-2526 (2008).

[13] G. Chintalapani, L. M. Ellingsen, O. Sadowsky, J. L. Prince, R. H. Taylor, "Statistical Atlases of Bone Anatomy: Construction, Iterative Improvement and Validation," Medical Image Computing and Computer Assisted Intervention (MICCAI), Part I. LNCS (4791), 499-506 (2007).

[14] W. Wein, A. Khamene, D. A. Clevert, O. Kutter, N. Navab, "Simulation and Fully Automatic Multimodal Registration of Medical Ultrasound," N. Ayache, S. Ourselin, A. Maeder (eds.) Medical Image Computing and Computer Assisted Intervention (MICCAI), Part I, LNCS 4791, 136-143. Springer, Heidelberg (2007).

[15] C. Nikou, B. Jaramaz, A. M. DiGioia, T. J. Levison, "Description of Anatomic Coordinate Systems and Rationale for Use in an Image-Guided Total Hip Replacement System," Medical Image Computing and Computer Assisted Intervention (MICCAI), LNCS 1935, 1188-1194 (2000).

[16] S. Gill, P. Mousavi, G. Fichtinger, D. Pichora, P. Abolmaesumi, "Group-wise Registration of Ultrasound to CT Image of Human Vertebrae," Proceedings of SPIE Medical Imaging, Visualization, Image-guided Procedures and Modeling, 7261, 726110 (2009).

[17] N. Hansen, and A. Ostermeier, "Completely Derandomized Self-Adaptation in Evolution Strategies," Evolutionary Computation, 9(2), 159-195 (2001).

[18] R. W. Prager, R. N. Rohling, A. H. Gee, L. Berman, "Rapid Calibration for 3-D Freehand Ultrasound," Ultrasound in Medicine and Biology 24(6), 855-869 (1998).

[19] P. J. Besel, N. D. McKay, "A method for registration of 3-D shapes," IEEE Transaction on Pattern Analysis and Machine Intelligence, vol 14, no. 2, 239-256 (1992).

[20] O. Kutter, R. Shams, N. Navab, "Visualization and GPU-Accelerated Simulation of Medical Ultrasound from CT Images," Computer Methods and Programs in Biomedicine, 2523-2526 (2009). 